\title{
THE EFFECT OF DIFFERENT FORCE LEVELS FOR MAXILLARY PROTRACTION ON THE UPPER INCIOSRS' POSITION IN GROWING CLASS III PATIENTS
}

\author{
Khaled M. Taha ${ }^{1}$, Farouk A. Hussien ${ }^{2}$, Ashraf A. El-Bedwehi ${ }^{3}$
}

\begin{abstract}
Objective: To evaluate changes in the anterior maxillary dentoalveolar segment concomitant with different levels of force application for maxillary protraction in growing skeletal Class III patients.

Subjects and Methods: The current study was conducted on 30 Class III patients (19 boys and 11 girls) with maxillary difficieny. The age of the recrutted sample ranged from 7-10 years. The patients were randomely allocated into three equal groups. The point of protraction force application varied among the three groups; Group A at the occlusal plane level, Group B at 20 $\mathrm{mm}$ above the occlusal plane level and Group $\mathrm{C}$ at the infraorbital foramen level. The duration of maxillary protraction was 8 months, the patients were instructed to wear the appliance for 16 hour/day with the applied 550gs protraction force. Before and after maxillary protraction $\mathrm{CBCT}$ images were used to evaluate the outcomes. Results: The vertical position of the upper central incisors varied significantly $(p<0.05)$ among the three studied groups as demonstrated by $\mathrm{U}_{1}-\mathrm{THP}$. In group A; the maxillary incisal edge moved away from the occlusal plane by $-2.20 \mathrm{~mm}$, in group B; there was non significant $(\mathrm{p}>0.05)$ change, however, in group C; it moved occlusally by the average $3.49 \mathrm{~mm}$. There was non significant $(\mathrm{p}>0.05)$ differences among the three studied groups regarding the changes in the saggital incisal position as well as the change in the incisor's inclination.
\end{abstract}

Conclusions: It could be concluded that the application of the protraction force in a level higher than the occlusal plane, is an efficient tool to control the vertical position of the maxillary incisors after protraction facemask therapy.

KEYWORDS: Protraction facemask, Multilevels protraction system, Class III malocclusion, Upper midface projection

\section{INTRODUCTION}

Skeletal Class III malocclusion is one of the most difficult conditions to correct in daily orthodontic practice. The continued post-treatment craniofacial growth is adding to the difficulty of this condition, especially when the mandible is diagnosed as the primary offending jaw. According to the literature, there is a great variability regarding the prevalence of Class III malocclusions across and within different populations. ${ }^{1}$ The incidence of Class III malocclusion among Egyptian population is at the rate of $11.8 \%^{(1,2)}$.

1. Assistant lecturer, Department of Orthodontics, Faculty of Dental Medicine (Boys, Cairo), Al-Azhar University.

2. Associate Professor\& Acting head, Department of Orthodontics, Faculty of Dental Medicine (Boys, Cairo), Al-Azhar University.

3. Professor, Department of Orthodontics, Faculty of Dental Medicine (Boys, Cairo), Al-Azhar University.

- Corresponding author: kh.taha@azhar.edu.eg 
Class III skeletal malocclusion may result from maxillary retrusion, mandibular prognathism, or combined maxillary retrusion and mandibular prognathism. According to Ellis and McNamara, they found in their sample that $65 \%$ to $67 \%$ of the observed Class III malocclusions were characterized by maxillary retrusion. Class III as considered a malocclusion in the sagittal dimension, it might also be associated with vertical as well as transverse discrepancies ${ }^{(3)}$.

There are several factors that should be considered when treating such cases; among them family history and genetics; severity of the problem; whether the problem is diagnosed in the midface, mandible, or combination; the age of the patient; patient compliance; in addition to the growth status of the patient ${ }^{(4)}$.

In the late 1960s, the Delaire protraction facemask was popularized to protract the maxilla. This appliance consists of a forehead cap and a chin cap that are connected together with bilateral square framework. There is a horizontal bar running in front of the mouth for elastic attachment. In 1983, Petit modified the Delaire mask by replacing the bilateral connecting square wire frame with a single vertical midline connecting bar. The horizontal running bar for elastic attachment is vertically adjustable in both designs to facilitate varying the point and direction of force application ${ }^{(5)}$.

It has been proven that the effects of the protraction facemask on the facial structures include; maxillary skeletal advancement, forward maxillary dentoalveolar movement, restraining the expected mandibular growth during the period of treatment, retrusion of the mandibular dentoalveolar segment as well as the increase in the lower anterior face height due to the counter clockwise rotation of maxilla and the backward rotation of mandible ${ }^{(6-8)}$.

According to Nanda, successful maxillary protraction could be done by controlling the protraction force variables such as the point of force application as well as the magnitude of the applied force. It was reported that the relationship between the point of protraction force application and the estimated center of resistance of the maxillary complex determines the direction of the maxillary rotation concomitant with forward maxillary displacement ${ }^{(9)}$.

It was also observed that the conventional tooth borne maxillary protraction headgears cause extrusion and anterior rotation of the anchor teeth, and upward and forward rotation of the maxilla ${ }^{(10-12)}$. According to Tanne et al the location of the center of resistance of maxilla is between the first and the second upper premolar root apexes ${ }^{(13)}$.

The spatial position of the maxillary incisors is of prime concern regarding the beauty of the smile and the attractiveness of the facial appearance. The anatomical location of the maxillary incisors enables them to act as valves that guard the postitive overget and help to achieve the long term stability. ${ }^{14,15}$

The counterclockwise rotation of the maxilla is usually accompanied with the lack of incisal show, increase in the incisal inclination in addition to open bite development especially in average and high angle cases. It was suggested that controlling the maxillary rotation would affect the postprotraction maxillary incisors position ${ }^{(17,18)}$.

Until recently, only three studies (18-20) have investigated the dentoskeletal effects of varying the point of protraction force application. None of these studies has used Cone Beam Computed Tomography (CBCT) to evaluate their outcomes nor applied the protraction force at a higher level above the nasal floor.

Therefore, the trigger to conduct this study was to answer the following question; does applying the protraction force in a level higher than the occlusal plan cause changes in the maxillary incisors' position after protraction facemask therapy in growing Class III patients? 


\section{SUBJECTS AND METHODS}

This randomized clinical trial was conducted on a sample of 30 Class III patients (19 boys and 11 girls) with age range from 7-10 years old. The sample size calculation was based data derived from previous studies ${ }^{(7,17)}$ for a standard deviation of $1.49 \mathrm{~mm}$ and a minimal intergroup difference of $2.14 \mathrm{~mm}$ to be detected, a sample of 27 patients was required to provide statistical power of $80 \%$ with an alpha of 0.05 .

The included patients met the following criteria: skeletal Class III malocclusion due to retruded maxilla and/or combined maxillary retrusion with mild mandibular prognathism $\left(\mathrm{ANB} \leq 1^{\circ}\right.$, Wits appraisal $<-2 \mathrm{~mm}$ ), concave profile with either edge to edge or anterior crossbite, no significant skeletal asymmetry, and no systemic diseases or congenital deformities. Only those subjects manifesting the features of pre-pubertal cervical vertebrae maturational stages 1 and 2 (initiation and acceleration) were included.(6)

This study was reviewed and approved from Department Ethics Committee, Faculty of Medicine for Boys, Al-Azhar University, Cairo, Egypt with registration number: orthod._10Med.Research_ Class III Malocclusion.Maxillary. Protraction. Growing.Pts._0000010 Additionally, the protocol of this study was registered in the ClinicalTrials.gov with the identifier: NCT04310267

After informed consent, the patients were randomly assigned into three equal groups according to the point of force application (Figure 1); Group A: occlusal level, where the point of force application was at the level of the occlusal plane with $30^{\circ}$ inclination ${ }^{(6,8)}$. Group B: nasal floor level, where the point of force application was at $20 \mathrm{~mm}^{(17)}$ above the occlusal plane. Group C: infraorbital level, where the point of protraction force application was approximately at the level of infraorbital foramen.

In order to relocate the point of force application in a higher positions as in groups $\mathrm{B}$ and $\mathrm{C}$ an intraoral to extraoral connecting device was used to overcome the anatomical limitations. This device is amodification of the standard headgear face bow (Denturum Company, Vogelberg 21BCH-4614 Hägendorf, Switzerland.) Maxillary protraction was preceded by rapid maxillary expansion for 10 days; 2 turns /day using bonded Hyrax (GH Wire Company, Hanover, Germany) rapid palatal expander( RPE). Extraoral Petit face mask (American Orthodontics 3524 Washington Avenue North America), used for extraoral anchorage, was linked to the intraoral RPE via extra oral elastics. $550 \mathrm{~g}$ per side ${ }^{(18)}$ were measured using force gauge to assure adequate orthopedic effect. Patients were instructed to wear the appliance at least 16 hour/ day for 8 months $^{(6)}$.

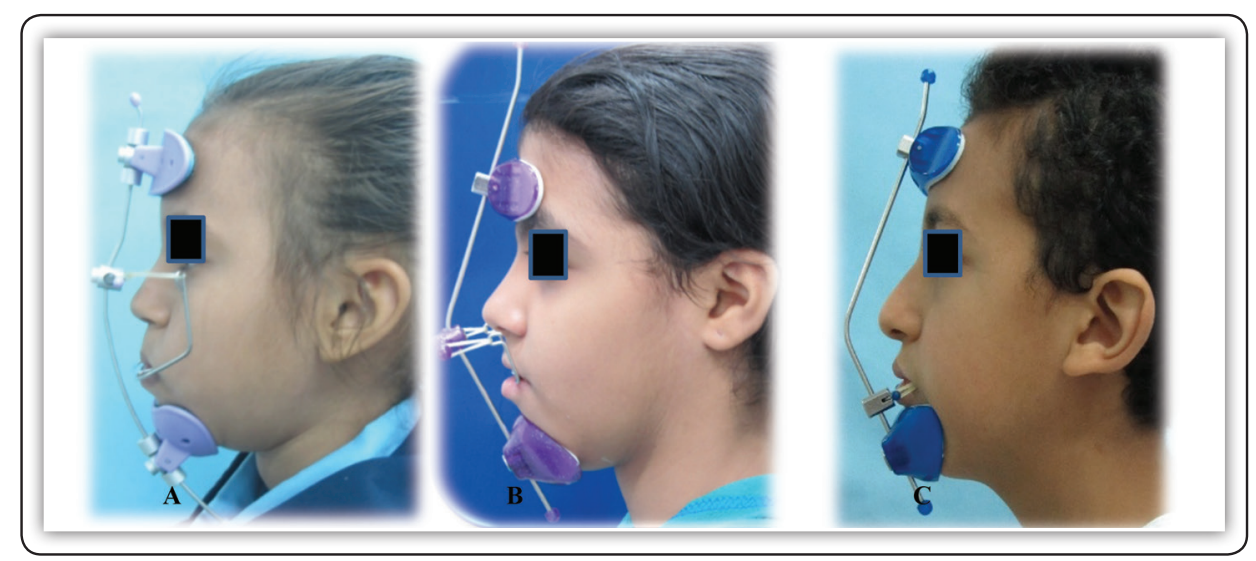

FIG (1) Showing extra oral photographvs illustrating different levels of the protraction forces; A: GroupA (occlusal level) , B: Group(Nasal floor level) B, C: Group C (infraorbital level) 


\section{Three dimensional assessment:}

The before and after maxillary protraction CBCT datasets were imported to the 3D on demand software (Cybermed co., Seoul, Korea), where 3D Ceph module was utilized for the analysis. Registration of both datasets was done using automatic registration, where certain area was identified as area with minimum anatomical changes at both scans, and then the software matches both volume accordingly. Due to unavoidable discrepancy in positioning patients at the CBCT machine, another reorientation is made to ensure that both datasets are now aligned to reference planes (Frankfurt horizontal and midsagittal planes).

After registration, and reorientation, the preprotraction dataset was the only activated at the tracing function, where the assigned landmarks were identified at the three planes. The software automatically calculated the linear and angular measurements (Table 1) as previously fed at the database. After this step, the post-protraction data set was then activated at the same volume configuration, two major tasks were then performed, the first one was identifying the previously located landmarks at certain coordinates at the volume, then moving each landmark from that coordinate to a new position according to the change in its anatomical position. The repositioning of landmarks was again identified, and corrected at the three reconstructed planes. After completion of landmark relocation process, the software again calculated the measurements. Both before and after measurement values were then extracted from the software in excel sheet format.

\section{Statistical Analysis}

Descriptive statistics were calculated for all measurements at $\mathrm{T} 1$ and $\mathrm{T} 2$ by using the Statistical Package for Social Science (version 23, SPSS, Chicago, Ill). Paired samples t-tests were used to determine the statistical significance of skeletal measurements between $\mathrm{T} 1$ and $\mathrm{T} 2$ for each group. T1-T2 changes among the three studied groups were assessed with one way ANOVA test. Statistical significance was tested at $\mathrm{P}<.05$.

\section{RESULTS}

The comparison of the baseline characteristics revealed non-significant differences among the three groups. Since the data sets of the studied outcomes were normally distributed, paired t-test was used to evaluate the treatment effect within each group. One way ANOVA test was used to evaluate the treatment among the three studied groups. The results of this study (Table 2) revealed statistically significant $(\mathrm{p}<0.05)$ difference among the three studied groups the changes in the vertical position

TABLE (1) Linear and angular measurements used to evaluate the change in the upper incisors' position and inclination after maxillary protraction

\begin{tabular}{|l|l|c|l|}
\hline \multicolumn{3}{|c|}{ Outcome measurement } & \multicolumn{1}{|c|}{ Definitions } \\
\hline Maxillary Incisors \\
\hline Position & Sagittal & $\mathrm{U}_{1}$-TVP $(\mathrm{mm})$ & The linear perpendicular distance between U1and TVP* \\
\cline { 2 - 5 } & Vertical & $\mathrm{U}_{1}$-THP $(\mathrm{mm})$ & The linear perpendicular distance between U1 and THP* \\
\hline Inclination & $\mathrm{U}_{1}-\mathrm{THP}\left({ }^{\circ}\right)$ & The posterior inferior angle at the intersection between U1 axial plane and PP \\
\hline
\end{tabular}

*TVP, True Vertical Plane

*THP, True Horizontal Plane
PP, Palatal Plane

$U_{1}$, Upper central Incisor (Incisal edge) 
TABLE (2) Comparison of the changes in the upper incisors' position and inclination after maxillary protraction among the three studied groups

\begin{tabular}{|c|c|c|c|c|c|c|c|c|c|c|c|}
\hline \multirow{2}{*}{\multicolumn{3}{|c|}{\begin{tabular}{|c|} 
Parameter (variable) \\
Maxillary incisors measurements
\end{tabular}}} & \multicolumn{2}{|c|}{$\begin{array}{c}\text { Group A } \\
\left(\mathbf{T}_{2-} \mathbf{T}_{1}\right)\end{array}$} & \multicolumn{2}{|c|}{$\begin{array}{c}\text { Group B } \\
\left(\mathbf{T}_{2-} \mathbf{T}_{1}\right)\end{array}$} & \multicolumn{2}{|c|}{$\begin{array}{c}\text { Group C } \\
\left(\mathbf{T}_{2-} \mathbf{T}_{1}\right)\end{array}$} & \multirow{3}{*}{$\begin{array}{c}\begin{array}{c}\mathbf{P} \\
\text { value }\end{array} \\
0.897\end{array}$} & \multirow{3}{*}{$\begin{array}{l}\text { Sig. } \\
0.419\end{array}$} & \multirow{3}{*}{$\begin{array}{l}\text { Sig. } \\
\text { NS }\end{array}$} \\
\hline & & & \multirow{2}{*}{$\begin{array}{c}\text { Mean } \\
3.02\end{array}$} & \multirow{2}{*}{$\begin{array}{l}\text { SD } \\
1.47\end{array}$} & \multirow{2}{*}{$\begin{array}{c}\text { Mean } \\
2.65\end{array}$} & \multirow{2}{*}{ SD } & \multirow{2}{*}{$\begin{array}{l}\text { Mean } \\
3.571\end{array}$} & \multirow{2}{*}{ SD } & & & \\
\hline Position & Sagittal & $\mathrm{U}_{1}-\mathrm{TVP}(\mathrm{mm})$ & & & & & & & & & \\
\hline & Vertical & $\mathrm{U}_{1}-\mathrm{THP}(\mathrm{mm})$ & -2.20 & 0.899 & 0.60 & 1.97 & 3.488 & 2.486 & 22.405 & 0.000 & $* * *$ \\
\hline \multicolumn{2}{|c|}{ Inclination } & $\mathrm{U}_{1}-\mathrm{THP}\left(^{\circ}\right)$ & 0.67 & 4.41 & -0.18 & 4.27 & 0.572 & 5.700 & 0.092 & 0.912 & NS \\
\hline
\end{tabular}

$T_{1}$ : before protraction, $T_{2}$ after protraction, $P$ value: Probability value, Sig. Significance, NS: None significant, *: significant

of the upper centeral incisors as demonstrated by U1-THP (mm). In group A; the incisal edge moved away from the occlusal plane by $-2.20 \mathrm{~mm}$, in group $\mathrm{B}$; there was non significant $(\mathrm{p}>0.05)$ change in the vertical incisal position. However, in group $\mathrm{C}$; maxillary the incisal edge, significantly $(\mathrm{p}<0.05)$, moved occlusally by the average $3.49 \mathrm{~mm}$. There was non significant ( $>0.05)$ differences among the three studied groups regarding the changes in the saggital incisal positionas well as the change in the incisor's inclination.

\section{DISCUSSION}

Tooth borne protraction facemask appliance is considered as the prime choice for the correction of Class III malocclusion with maxillary deficiency. The protraction facemask applies an anterior force on the circum maxillary sutures and stimulates bone formation at the sutural areas. It assists to correct the maxillary skeletal retrusion, maxillary dentoalveolar retrusion as well as the decrease in the lower face height.

There are some effects concomitant with the use of protraction facemask that might be undesrirable in certain clinical situations. One of these undesirabl effects,especially in cases of Class III with openbite tendency, is the lack of incisal show due to the counterclockwise rotation of the maxilla. So, the aim of this study was directed toward testing the hypothesis of varying the point of force application in order to overcome this side effect.

The protocol for this study was published online in order to avoid selective reporting or reporting bias. The current study included 30 Egyptian children of both genders with age range from $7-10$ years old. The early treatment at this age, according to literature, will help to achieve the maximum skeletal changes with the minimum dental compensations ${ }^{(19)}$. It was reported that there is no existing significant sexual dimorphism in Class III malocclusion before age of 13 years old therefore, no gender restriction in the selection of children was applied in this study ${ }^{(20)}$.

The selected patients were randomly allocated into three equal groups using online software (Random Allocation Software) in order to equally distribute the eligible patients among the three groups without bias. The comparison of the baseline characteristics of the subjects among the three groups revealed a non-significant differences in the all the studied outcomes, assuring the adequate method of randomization.

Allocation concealment was done by using white opaque envelopes, each envelope contained the random number and its allocation group. These envelopes were kept in a box at the orthodontic department secretary's office. The secretary of the department used to deliver the allocation envelop for each enrolled patient to the operator. 
Blinding during intervention procedures for both the operator and the patient was impossible. Meanwhile, blinding during data collection and analysis was possible. Data collection from patients' record (CBCT analysis) and statistical analysis was done by two persons other than the two supervisors and the principle operator in order to minimize data collection and management bias.

The maxillary protraction system set up in this study included the use of a Petit facemask attached to a fixed intra-oral bonded rapid palatal expander. Petit type facemask was chosen as it is relatively small in size which makes it more appealing to the child in the view of the esthetic concerns.

The treatment started with 10 days of RPE before applying protraction force in all groups to mechanically disarticulate the surrounding sutures and to release the inflammatory mediators that are important for facilitating maxillary displacement. It was also reported by Hass that maxillary expansion by itself can cause anterior maxillary displacement and slight increase in the vertical dimension that might help in the correction of mild class three cases. ${ }^{(21)}$ The occlusal coverage (splint) was incorporated in the design of the RPE for three reasons; first to disarticulate the occlusion that facilitates protraction and removes interferences, second is to counteract the extrusive effects of expansion and protraction and finally to help in splinting the maximum number of teeth as one unit that would increase the skeletal effect.

The maxillary skeletal effects of each point of force application were analyzed using CBCT due to its high accuracy and precision, sensitivity and specificity, as well as absence of image magnification. The comparison of the treatment effects in each group was made in a way previously described by several investigators ${ }^{(14)}$.

The results of this study, demonstrated a significant $(\mathrm{P}<0.05)$ positive response to facemask therapy at different levels of the force application regarding the amount of anterior maxillary displacement with non-significant $(\mathrm{P}>0.05)$ differences among the three studied groups.This finding is in accordance with what have been previously reported in the literature ${ }^{5-8}$.

The change of the upper incisors' vertical position varied significantly $(\mathrm{P}<0.05)$ among the three studied groups. In the occlusal plane group, the incisal edge moved away from the occlusal plane, while in the infraorbital group the opposite was true. These findings might be attributed to the counterclockwise and clockwise rotations of the maxilla in both the occlusal and infraorbital groups, respectively. The observed non-significant change in the vertical position in of the upper incisors in the nasal floor group could be ascribed to the tendency for the bodily movement of the maxilla in this group.

Conversaly, to what was reported in literature $^{(19,20)}$, the treatment changes in the sagittal position as well as the inclination of the maxillary incisors revealed non significant differences among the three groups. It was reported that the maxillary incisors retruded and retroclined after application of the protraction force higher than the occlusal level ${ }^{(19,20)}$. This contradiction might be attributed to the difference in the appliance design as well as the age of treated samples.

\section{CONCLUSIONS}

It could be concluded that the application of the protraction force in a level higher than the occlusal plane, is an efficient tool to control the vertical position of the maxillary incisors after protraction facemask therapy.

\section{CLINICAL IMPLICATIONS}

In class III patients with open bite tendency, applying the maxillary protraction force near to or at the level of the infraorbital foramen will not only help to correct the saggital problem but also assists in correcting the vertical problem with minimal side effects. 


\section{REFERENCES}

1. Hardy, D., Cubas, Y. and Orellana, M. Prevalence of angle Class III malocclusion: A systematic review and metaanalysis. Open J. Epidemiol. 2012; 2, 75-82.

2. El-Mangoury NH, Mostafa YA. Epidemiologic panorama of dental occlusion. Angle Orthod. 1990;60:207-14.

3. Ellis 3 E, McNamara Jr JA. Components of adult Class III malocclusion. Int J Oral Max Surg. 1984;42:295-305.

4. Begum Khan M, Karra A. Early Treatment of Class III Malocclusion: A Boon or a Burden? Int J Clin Pediatr Dent. 2014;7:130-6.

5. Shanker S, Ngan P, Wade D, Beck M, Yiu C, Hägg U, Wei SH. Cephalometric A point changes during and after maxillary protraction and expansion. Am J Orthod Dentofac Orthop. 1996;110:423-30.

6. Ngan P, Yiu C, Hu A, Hägg U, Wei SH, Gunel E. Cephalometric and occlusal changes following maxillary expansion and protraction. Euro J Orthod. 1998;20:237-54.

7. Ngan PW, Hagg U, Yiu C, Wei SH. Treatment response and long-term dentofacial adaptations to maxillary expansion and protraction. Seminars in orthodontics $1997 \mathrm{D}$ (Vol. 3, No. 4, pp. 255-264). WB Saunders.

8. Ngan P, Wilmes B, Drescher D, Martin C, Weaver B, Gunel E. Comparison of two maxillary protraction protocols: tooth-borne versus bone-anchored protraction facemask treatment. Prog in orthod. 2015;16:26

9. Kambara T. Dentofacial changes produced by extraoral forward force in the Macaca irus. Am J Orthod. 1977; 71:249-77.

10. Yepes E, Quintero P, Rueda ZV, Pedroza A. Optimal force for maxillary protraction facemask therapy in the early treatment of Class III malocclusion. European journal of orthodontics. 2014 ;36:586-94.

11. Nardoni DN, Siqueira DF, Cardoso MD, Capelozza Filho L. Cephalometric variables used to predict the success of interceptive treatment with rapid maxillary expansion and face mask. A longitudinal study. Dental Press J Orthod $2015 ; 20: 85-96$.

12. Nanda R. Protraction of maxilla in rhesus monkeys by controlled extraoral forces. Am J Orthod. 1978 Aug 1;74:121-41.
13. Celikoglu M, Yavuz I, Unal T, Oktay H, Erdem A. Comparison of the soft and hard tissue effects of two different protraction mechanisms in Class III patients: a randomized clinical trial. Clin Oral Investig. 2015;19:2115-22.

14. Ge YS, Liu J, Chen L, Han JL, Guo X. Dentofacial effects of two facemask therapies for maxillary protraction Miniscrew implants versus rapid maxillary expanders. Angle Orthod. 2012;82:1083-91.

15. Turley PK. Managing the developing Class III malocclusion with palatal expansion and facemask therapy. Am J Orthod Dentofac Orthop 2002;122:349-52.

16. Tanne K, Sakuda M. Biomechanical and clinical changes of the craniofacial complex from orthopedic maxillary protraction. Angle Orthod. $1991 ; 61: 145-52$.

17. Tanne K, Hiraga J, Sakuda M. Effects of directions of maxillary protraction forces on biomechanical changes in craniofacial complex. Eur J Orthod. 1989;11:382-91.

18. Nanda R. Biomechanical and clinical considerations of a modified protraction headgear. Am J Orthod. 1980;78:125-39. A

19. Alcan T, Keles A, Erverdi N. The effects of a modified protraction headgear on maxilla. Am J Orthod Dentofacial Orthop. 2000;117:27-38.

20. Keles A, Tokmak EÇ, Erverdi N, Nanda R. Effect of varying the force direction on maxillary orthopedic protraction. Angle Orthod. $2002 ; 72: 387-96$.

21. Baccetti T, McGill JS, Franchi L, McNamara Jr JA, Tollaro I. Skeletal effects of early treatment of Class III malocclusion with maxillary expansion and face-mask therapy. Am J Orthod Dentofac Orthop. 1998;113:333-43.

22. Franchi L, Baccetti T, McNamara JA. Postpubertal assessment of treatment timing for maxillary expansion and protraction therapy followed by fixed appliances. Am J Orthod Dentofac Orthop. 2004;126:555-68.

23. Yatabe M, Garib DG, Faco RA de S, de Clerck H, Janson G, Nguyen T, Bone-anchored maxillary protraction therapy in patients with unilateral complete cleft lip and palate: 3-dimensional assessment of maxillary effects. Am J Orthod Dentofac Orthop. 2017;152:327-35. 